\title{
Cittadinanza multilivello, nuovi rischi sociali e welfare regionale
}

\author{
di Luis Moreno* e Alessandro Gentile**
}

\section{Sommario}

L'articolo propone un'analisi concettuale dei fondamenti del Modello Sociale Europeo come sistema di welfare multilivello. In particolare, espone l'importanza della sussidiarietà amministrativa tra i diversi livelli della governance europea come un elemento centrale della cittadinanza sociale. Con questa espressione viene definita l'articolazione strategica tra legittimità sovra-nazionale e responsabilità sub-statale che ottimizza l'implementazione di politiche pubbliche. L'impatto dei cosiddetti “nuovi rischi sociali” (NSR) dipende dalle possibilità di sviluppo sociale, strutturale ed istituzionale delle comunità locali. In questo senso, l'autonomia fiscale a livello regionale è un aspetto centrale per garantire politiche redistributive e di solidarietà. Partendo da queste riflessioni, nell'ultima sezione si valuta come la governance multilivello possa rafforzare la cittadinanza sociale europea grazie all'interscambio di esperienze positive e innovative tra diversi stati membri, specialmente nell'attuale congiuntura di instabilità economica internazionale.

Classificazione: $H 790$

Parole Chiave: Governance, Unione Europea, Sussidiarietà, Politiche sociali, Comunità locali.

\section{Multilevel Citizenship, New Social Risks and Regional Welfare}

\begin{abstract}
In this paper we carry out a conceptual review of European Social Model's basic issues as a multilevel welfare system. We outline administrative subsidiarity between different levels of European governance as one of the main pillar of social citizenship. With such expression we define the strategic configuration between transnational legitimacy and sub-state accountability in the provision of public policies. The impact of the so-called "new social risks" (NSR) relies on the social, structural and institutional development of local communities. According to that, financial autonomy at a regional level is a key resource for policy innovation together with the concomitants issues of redistribution and solidarity. Starting from these issues, the concluding section of the paper puts forward the idea that the advancement of social citizenship in the European Union may be best achieved through the interchange of positive and innovative experiences among and within member states, especially in the current international situation of economic instability.

JEL Classification: $H 790$

Keywords: Governance, European Union, Subsidiarity, Social Policies, Local Communities.
\end{abstract}

* Professore di Ricerca dell’Instituto de Políticas y Bienes Públicos, del Consejo Superior de Investigaciones Científicas, IPP-CSIC, Calle Albasanz 26-28, Madrid, E-mail: luis.moreno@csic.es.

** Professore di Sociologia del Dipartimento di Sociología I (Cambio Social) dell’Universidad Complutense de Madrid, Campus de Somosaguas, Pozuelo de Alarcón, Madrid. E-mail: alessandro.gentile@cps.ucm.es.

argomenti, 37/2013 


\section{Introduzione: transnazionalizzazione e sussidiarietà}

Dopo la Seconda Guerra mondiale, i sistemi di welfare si sono sviluppati principalmente con un modello centralista di policy-making, trattando i concetti di uguaglianza e omogeneità come sinonimi. D’accordo con questa prospettiva, la copertura dei diritti sociali dei cittadini dovrebbe essere eseguita da un'amministrazione unitaria e centralizzata di servizi pubblici. Negli ultimi decenni, l'importanza dello stato-nazione come responsabile dei sistemi moderni di previdenza sociale ha progressivamente lasciato il passo a istituzioni sovra-nazionali e sub-nazionali per lo sviluppo del welfare. Da un lato, la transnazionalizzazione ha favorito il potere di influenza strategica di nuove entità politiche a livello internazionale; dall'altro, parallelamente, le comunità territoriali (regionali e locali) hanno rafforzato la loro domanda di sussidiarietà per avere maggiori prerogative con rispetto alla offerta e alla amministrazione dei servizi di welfare. Queste due dinamiche hanno messo in discussione il centralismo esercitato dagli stati-nazione, nella forma di "comando e controllo" (command-andcontrol), con iniziative e programmi territorialmente differenziati di politiche sociali.

I processi di integrazione continentale - come l'europeizzazione - e di decentramento dei poteri fanno si che le politiche vengano considerate aldilà degli interessi nazionali di ogni singolo stato. È possibile affermare che nelle democrazie a capitalismo avanzato l'era della centralizzazione nazionale sia stata seguita da un periodo di decentralizzacione (Marks et al., 2008) $)^{1}$. Nel presente articolo, i riferimenti all'Unione Europea ci aiutano ad illustrare meglio tali processi.

Il consolidamento del terzo ciclo di cittadinanza sociale teorizzato da Marshall $^{2}$ si dovrebbe raggiungere nella quarta fase di costruzione sistemica dell'Europa come comunità economica, politica e sociale ${ }^{3}$. Da un

\footnotetext{
${ }^{1}$ Gli autori ringraziano la European Science Foundation (Eurocores-Hum-VIB) e il Ministero spagnolo di Innovazione e Scienza, entrambi sponsor del progetto Welfare Attitudes in a Changing Europe (MICINN, CSO2008-02874) e del progetto Solidaridad familiar, cambio actitudinal y reforma del Estado de Bienestar en España: el familismo en transición (MICINN, CSO2011-27494) le cui attività di studio e di ricerca hanno reso possibile la realizzazione di questo articolo.

${ }^{2} \mathrm{Si}$ fa riferimento alla fase storica successiva una volta che si concretizza la cittadinanza politica e sociale (Marshall, 1950 e 1965). Marshall spiega il conflitto esistente tra l'uguaglianza politica e i diritti sociali, da un lato, e il mercato capitalista e la classe sociale, dall'altro, in una prospettiva dinamica piuttosto che secondo una teoria evoluzionista.

${ }^{3}$ Stein Rokkan (1921: 79) si è concentrato sulle relazioni tra la struttura interna e i limiti esterni per comprendere meglio come la formazione militare e amministrativa dello stato, la
} 
punto di vista istituzionale e territoriale, questa realtà si configura nell'ambito della caratteristica stratificazione interna dell'Unione Europea (Bartolini, 2005). Infatti, l'apertura delle strutture di governance sovranazionale si sta realizzando attraverso interazioni di tipo multilivello che hanno interessato soprattutto quegli attori, quei poteri regolatori e quei network di policy le cui competenze erano state tradizionalmente limitate nell'ambito degli stati-nazione. Attualmente, più della metà della legislazione che influenza la vita degli europei è emanata dall'Unione Europea. Come contesto politico di riferimento, l'Unione è composta da dinamiche interconnesse che definiscono in larga misura la sovranità formale di ciascun stato membro (Piattoni, 2010).

Ciononostante, non si può immaginare la formazione degli Stati Uniti d'Europa come il risultato finale inevitabile della europeizzazione. Secondo un paradigma neo-funzionalista, qualsiasi tipo di progresso universale richiede un modello integrato, così come è possibile osservare nei casi di assimilazione culturale e di formazione identitaria complessa, per esempio nel caso del melting-pot americano o della cosiddetta "insalatiera" multiculturale. In maniera alternativa, la definizione di norme comuni in Europa potrebbe essere considerata come il risultato dell'adattamento di una larga diversità storica e culturale nel mosaico dei popoli che abitano il vecchio continente. A questo proposito, la cittadinanza multilivello è un concetto chiave che deve essere interiorizzato pienamente dagli europei (Berg, 2007).

La domanda di una maggiore sussidiarietà territoriale è stata promossa da istituzioni di livello intermedio, come regioni e municipi. Si tratta di autorità locali che spesso non sentono come necessaria l'ingerenza di burocrazie centraliste nell'esercizio della propria autonomia e delle proprie iniziative politiche. Inoltre, le comunità politiche sub-statali godono di una sicurezza economica e politica che gli viene offerta da organismi sovranazionali, come appunto l'Unione Europea, in un contesto di sostenuta transnazionalizzazione (Keating, 2001; Moreno e McEwen, 2005).

La sussidiarietà favorisce un ambito di partecipazione sociale e di decisione politica che sia il più possibile decentralizzato e vicino alla cittadinanza ${ }^{4}$. Le élite politiche di quegli stati membri che sono riluttanti a

costruzione culturale di una nazione e la diffusione politica delle masse democratiche si intersecano tra loro (Flora et al., 1999). Lo sviluppo del welfare è l'ultima fase che Rokkan utilizza per definire un mappa tipologico-topologico d'Europa.

${ }^{4}$ Il principio di sussidiarietà è sancito nel Trattato di Maastricht del 1992 mediante due diverse definizioni: la prima è sostanziale, appare nel preambolo e nell'Art.1, ed esprime 
una maggiore transnazionalizzazione hanno assunto tenacemente questo principio come una garanzia per preservare il potere di intervento centralizzato nelle rispettive arene di governo. In questo modo, l'essenza della sussidiarietà viene posizionata a un livello che non sia inferiore al nazionale ${ }^{5}$.

In questo articolo elaboriamo l'idea della "cittadinanza multilivello" come insieme di impegni collettivi che favoriscono la legittimità sovranazionale e la responsabilità democratica sub-statale per implementare le politiche sociali. La nostra analisi si concentra principalmente su quest'ultimo aspetto. Al contempo, dedichiamo particolare attenzione alle aspirazioni delle regioni e di altri livelli di governance locale per promuovere la cittadinanza sociale. Realizziamo una breve rassegna teorica dei diversi impegni assunti dai cittadini in contesti istituzionali di tipo multilivello e transnazionale. $\mathrm{Ci}$ occupiamo, quindi, delle possibili configurazioni di welfare che si sviluppano a livello sub-statale per controllare e prevenire l'impatto dei cosiddetti "nuovi rischi sociali" (NSR). In questo senso, l'autonomia fiscale a livello regionale è un aspetto centrale per garantire politiche redistributive e di solidarietà. Partendo da queste riflessioni, nell'ultima sezione dell'articolo valutiamo come la governance multilivello possa rafforzare la cittadinanza sociale europea grazie all'intercambio di esperienze positive ed innovative tra diversi stati membri, specialmente nell'attuale congiuntura di grave instabilità economica internazionale.

\section{Identità dei cittadini, appartenenza multipla e politica sociale}

un'ampia intesa politica e concettuale sui criteri di prossimità e proporzionalità; la seconda è di carattere procedurale, viene presentata nell'Art.5 (originariamente 3b), e ha avuto una implementazione legale piuttosto problematica (van Hecke, 2003).

5 I dibattiti nell’Unione Europea sul cosiddetto "guardiano della sussidiarietà" (subsidiarity watchdog) illustrano le difficoltà esistenti per conciliare gli interessi nazionali e regionali. Questi due livelli politici ed amministrativi hanno avviato una disputa sull'opportunità o meno di introdurre una procedura addizionale prima dell'entrata in vigore di una misura legislativa europea che confliggerebbe con i poteri acquisiti in ciascun ambito di governo. Tale procedura stabilisce che l'applicazione di una parte della legislazione europea verrebbe sospesa, ed eventualmente presentata alla Corte Europea per decidere sui principi ultra vires, se una qualificata minoranza di stati membri o una significativa minoranza di "regioni partner" (partner regions, cioè quelli con poteri legislativi) non fossero d'accordo. Si tratta, quindi, di misure di design costituzionale che sarebbero di estrema importanza nella ristrutturazione delle disposizioni di welfare (Moreno e Palier, 2005). 
Stato e nazione sono concetti trattati spesso come sinonimi nella modernità. Tuttavia, alla fine del ventesimo secolo, la loro effettiva congruenza è stata accertata solamente in una dozzina di paesi nel mondo (Hobsbawm, 1996). Generalmente, gli stati sono multinazionali perché sono composti da una varietà di comunità politiche. Quest'ultime devono intendersi come gruppi di persone governate e rappresentate dalle strutture di un determinato sistema politico, sia esso nazionale o regionale (Easton, 1965). In un contesto di governance multilivello, le comunità politiche possono estendersi dal livello locale al sovra-nazionale, indipendentemente dei requisiti di sovranità (Hooghe e Marks, 2001; Loughlin, 2007).

L'identificazione con lo stato-nazione è continuata ad essere comunemente considerata dai suoi integranti come la condizione minima indispensabile per definire lo status di cittadinanza. Malgrado ciò, negli ultimi venti anni, l'esclusività identitaria degli stati-nazione è stata messa apertamente in discussione, fino a diventare problematica. Oltre ad essere erosa dalle forze della globalizzazione, questa identità viene anche esposta a processi di frammentazione, competizione e sovrapposizione di diversa natura, specialmente in quei sistemi di governo caratterizzati da una composizione molteplice e plurale. Per quanto riguarda il nostro caso di studio, la cittadinanza dell'Unione Europea non rimpiazza la cittadinanza nazionale, bensì la complementa. Di conseguenza, assistiamo a un notevole rafforzamento delle identità sub e sovra-statali (Moreno, 1999). Come già si era avuto modo di osservare negli anni novanta, poco più di un terzo delle persone intervistate per il World Survey Value considerava che l'identità più importante per loro era la nazionale, mentre il 50\% preferiva l'identità locale o regionale (Norris, 2000).

Con l'autonomia territoriale, la federalizzazione ed il conseguente trasferimento di competenze dal governo centrale alle autorità locali, si cerca di offrire una risposta adeguata agli stimoli provenienti dai diversi sistemi amministrativi locali. Tali realtà comprendono comunità politiche regionali con identità, storia, lingua e tradizione differenti, che spesso si riflettono in una varietà di partiti, e dunque in una amplia pluralità di canali di rappresentanza in vista di interessi specifici. Sin dalla metà degli anni ottanta, un ampio riscontro empirico e statistico ha sostenuto l'ipotesi conosciuta come "questione Moreno": è stata dimostrata la congruenza - in varie forme e gradi - tra l'attaccamento identitario ad entrambi i livelli, statale e sub-statale. I casi di identità duale e di identità multiple evidenziano questi processi attraverso cui i cittadini esprimono lealtà ed aspirazioni politiche condivise grazie alla varietà dei livelli di riferimento. 
L'enfasi sulle identità e sui sistemi di governo territoriali evidenziano un grado significativo di diversità interna che non si associa solamente alla distinzione singolare o caratteristica, ma anche alle relazioni di democrazia, reciprocità e cooperazione esistenti (Linz, 1997; Gagnon e Tully, 2001; Moreno, 2005).

Nell'Unione Europea, la cittadinanza può essere considerata come il prodotto di identità integrate (nested identities) formatesi in contesti dove si intersecano tutte le varie relazioni politiche mantenute dai cittadini (a livello sovra-statale, statale, e sub-statale) (Faist, 2001; Díez Medrano e Gutiérrez, 2003; Bruter, 2005). Queste molteplici forme identitarie di cui sono titolari gli europei si inseriscono in un variabile continuum di appartenenze ed affinità territoriali radicate sui valori dei diritti umani e della solidarietà. Di fatto, i diritti civili e politici individuali fanno sempre più riferimento al livello regionale degli stati membri dell'Unione Europea. Come se non potesse essere altrimenti, l'esercizio di tali diritti è sfociato in una cittadinanza sociale sempre più consolidata in una dimensione locale (Jeffery, 2009).

Pertanto, la cultura civica europea può essere intesa come una "appartenenza multipla” (compound civility) ${ }^{6}$, dove l'offerta di policy deve essere in sintonia con una configurazione istituzionale di welfare con caratteristiche diverse, funzionali e territoriali. La pluralità di appartenenze identitarie suppone, da un lato, la possibilità di una affiliazione molteplice a regioni e a nazioni europee (con o senza stato) e, dall'altra, integra anche una linea comune - in molti casi mista e intrecciata - che definisce l'assiologia centrale del Modello Sociale Europeo. Il MSE si afferma come un sistema di valori condiviso, che rende possibile la solidarietà e legittima la redistribuzione di risorse e di opportunità in tutti i regimi di welfare europei $^{7}$. Durante il secolo passato, il Welfare State (una "invenzione"

\footnotetext{
${ }^{6}$ La civiltà (civility) potrebbe essere associata non solamente al concetto di cittadinanza ma anche all'idea di patriottismo. In un senso più ampio, il patriottismo appella a un attaccamento alle istituzioni e ai valori di una determinata comunità politica in modo simile all'affinità esistente tra i membri di una famiglia. L'aggiunto constitutional, come in constitutional patriotism è un elemento generalmente addizionale ma spesso confuso con l'ideologia del nazionalismo. Il patriota può essere leale alla lingua, alla storia e alla cultura del proprio paese. Tuttavia, questo paese potrebbe essere integrato in una comunità politica più estesa (come per esempio il caso della Scozia e della Gran Bretagna) (Moreno, 2006).

${ }^{7}$ Questa proposta entra in contrasto con altri modelli, come quello statunitense, per quanto riguarda i valori che caratterizzano alcuni aspetti della vita sociale. Si pensi ad esempio al tipo di pressione fiscale: diversamente da quanto accade nel vecchio continente, negli Stati Uniti la maggioranza degli intervistati nelle inchieste nazionali dichiara che ricchi
} 
europea) ha incorporato istituzionalmente i valori di equità e compassione, lasciando aperta la questione riguardo alla possibilità di garantire la giustizia sociale oltre lo stato-nazione. A questo proposito, si sostiene che le identità e le culture locali potrebbero limitare la solidarietà dei welfare nazionali. A queste accuse si risponde da diversi punti di vista - anche da quelle prospettive vicine alla teoria democratica - con l'argomento che l'identità collettiva continua ad essere una premessa indispensabile per legittimare le società industrialmente avanzate (Dahl, 1989; Held, 1991; Giner, 1994; Archibugi et al., 1998).

Lo sviluppo del welfare europeo sovra-nazionale, e del suo corollario di politiche sociali top-down, è poco probabile nel prossimo futuro. Tutti i livelli amministrativi (nazionale, regionale e locale) contribuiranno a modellare le aspettative, le attitudini e le percezioni dei cittadini con i loro input. Le forze capitaliste già da tempo sono attente a tutte le opportunità disponibili per calibrare le proprie attività sul territorio con l'intenzione di ottimizzare gli investimenti. Questi processi di assestamento territoriale in un contesto più dinamico e permeabile per le iniziative imprenditoriali e finanziarie influenzeranno in maniera diretta gli standard vitali dei cittadini nelle rispettive regioni e località di residenza (Somerville, 2004; Moreno, 2007; Ferrera, 2008).

Per le scienze sociali non è facile valutare e indicare quando il livello di benessere dei cittadini è "insoddisfacente" in una regione $\mathrm{o}$ in una comunità locale incapace di autosostenersi ed autoregolarsi. In queste circostanze gli incentivi degli attori e delle istituzioni nazionali e transnazionali possono risultare decisivi per il mantenimento delle condizioni minime di sussistenza e di benessere, sebbene tali ingerenze a volte possano anche favorire alcune anomalie politiche come il clientelismo verticale e le pratiche di concorrenza sleale (social dumping).

La sfera della politica sociale è molto influenzata dalle culture e dagli stili di vita in un ambito locale, mentre ha meno a che vedere con la prospettiva omogenea e centralizzata di una entità sopra-nazionale (Moreno, 2003; Ferrera, 2005). Negli ultimi decenni, le regioni hanno riaffermato le proprie identità politiche realizzando interventi concreti per lo sviluppo del welfare. In questo senso, hanno svolto un ruolo efficace per il progresso della cittadinanza sociale, soprattutto nell'ambito delle politiche attive per il lavoro, dell'assistenza sociale, dei servizi di attenzione primaria alle persone e delle politiche di compensazione delle

e poveri dovrebbero pagare la stessa proporzione di tasse sui loro salari (Lewis-Beck et al., 2008). 
reti di protezione sociale (Fargion, 2000; Arriba e Moreno, 2005 e 2010; Kazepov, 2008; Del Pino e Van Ryzin, 2013). Questi interventi aumentano grazie anche alla crescente implicazione del Terzo Settore e delle ONG, alla sempre maggiore offerta di servizi al welfare pubblico da parte di attori for-profit, e al rafforzamento di programmi sociali offerti dalle imprese (corporate welfare). Stiamo parlando di tutta una serie di sviluppi che, con il "residuale" ruolo di regolamentazione da parte dei governi, confluiscono in un welfare mix come aggregato di preferenze per conciliare le differenze ideologiche, sostenere la crescita economica e favorire la coesione sociale. L'affermazione dei "nuovi rischi sociali” (NSR) mette a prova le capacità collaborative di tutti questi attori per ottimizzare l'azione e la struttura del welfare mix. Nella prossima sezione, ci occupiamo di come il welfare substatale, a un livello regionale, riesce a mantenersi in questo nuovo scenario.

\section{Nuovi rischi sociali e welfare regionale}

Durante gran parte del ventesimo secolo, il principale obiettivo della istituzionalizzazione del welfare è stata la promozione dei diritti sociali nelle democrazie industriali. Anche dopo l'entrata nel nuovo millennio, la cittadinanza sociale in Europa continua ad essere una priorità legittima per le democrazie post-industriali, malgrado i cambiamenti di ruoli e funzioni che produttori e consumatori di welfare hanno dovuto realizzare in contesti internazionali in continuo mutamento a causa del nuovo ordine globale.

Le modifiche strutturali più significative si sono prodotte nelle democrazie avanzate a seguito della diffusione dei "nuovi rischi sociali". Questi NSR sono associati alla transizione a una società post-industriale (post-fordista) e includono quattro elementi principali: (1) la maggiore partecipazione delle donne al mercato del lavoro formale; (2) l'aumento delle persone anziane e dipendenti; (3) la crescita dell'esclusione sociale per i lavoratori con bassi livelli di qualificazione; (4) l'espansione di servizi privati "irresponsabili" con la contemporanea deregolazione dei loro omologhi nel settore pubblico (Esping-Andersen et al., 2002; TaylorGooby, 2004).

Come conseguenza dei NSR, i gruppi vulnerabili presentano nuove necessità con rispetto a quattro questioni particolarmente attuali come: (i) la conciliazione tra il lavoro salariato e le responsabilità di cura e mantenimento nella sfera familiare e domestica; (ii) l'attenzione a parenti e anziani non autosufficienti, o l'invecchiamento senza una rete familiare di sostegno; (iii) la mancanza delle competenze necessarie per avere accesso a 
un posto di lavoro sicuro e adeguatamente remunerato, oppure un tipo di formazione ormai obsoleta che non è possibile attualizzare per la mancanza di un sistema di apprendimento permanente; (iv) l'impiego di risorse private che siano in grado di supplire a una pensione insicura 0 insufficiente o a dei servizi poco soddisfacenti (Bonoli, 2005; Armingeon e Bonoli, 2007).

La crescita incontrollata ed il superamento strategico (crowding out) degli interventi pubblici di Welfare destinati ai "vecchi rischi sociali" (analfabetismo, vecchiaia, malattia o disoccupazione) hanno incoraggiato le regioni ad esplorare nuovi interventi che riguardassero più concretamente $\mathrm{i}$ NSR. Alcuni difensori del centralismo statale e sostenitori della ortodossia neo-classica insistono da decenni sulla irreversibilità della riduzione del welfare. Dal loro punto di vista, l'esplosione delle domande di copertura sociale ha provocato una implosione delle decisioni da parte dei governi centrali che hanno dovuto fronteggiare un numero crescente di restrizioni politiche. In una prospettiva neo-marxista, entrambi i processi di legittimazione del sistema capitalista e di erosione dei meccanismi di crescita che esso stesso favoriva sono arrivati a un punto di saturazione ${ }^{8}$. Negli ultimi anni, comunque, risulta più preciso parlare di contenimento della spesa sociale (cost containment) messo in atto dalle istituzioni finanziarie centrali in Europa piuttosto che di riduzione dei diritti sociali e dei programmi di welfare (retrenchment) come avviene attualmente. Ciò significa, quindi, che la previdenza sociale è stata preservata grazie a sistemi di tassazione e assicurazione altamente legittimati (Kuhnle, 2000; Pierson, 2001; Taylor-Gooby, 2001).

L'insieme dei NSR menzionati in precedenza suppone nuovi aggiustamenti per i sistemi di Welfare. Da un lato, si questiona l'articolazione del welfare mix in contesti caratterizzati dalla cittadinanza multilivello e, dall'altro, si dibatte su come combinare i nuovi ed i "vecchi" impegni assunti dai sistemi di previdenza sociale. Con l'affermarsi di uno scenario così complesso, il ruolo delle istituzioni transnazionali e le iniziative dei governi regionali e locali hanno assunto una rilevanza politica crescente. In particolare, le amministrazioni sub-statali hanno contribuito a una moderata espansione del welfare per rispondere ai NSR, in modo da dare un impulso alla cittadinanza sociale. In diversi stati membri dell’Unione Europea alcune regioni si sono attivate per l'innovazione

${ }^{8}$ Il "racconto di O’Goffe” si riferisce alle posizioni di O’Connor (1973), Gough (1979) e Offe (1984) secondo i quali esiste una contraddizione insuperabile per il mantenimento di un Welfare State solidale ed inclusivo. 
politica soprattutto nei settori dell'assistenza e nelle reti di protezione sociale. Il piano regionale contro la povertà nel Paesi Baschi o l'assistenza gratuita per gli anziani in Scozia sono esempi concreti di queste iniziative territoriali.

Se torniamo indietro nel tempo, alla fine degli anni ottanta, ricordiamo che il governo regionale basco introdusse un piano contro la povertà stabilendo un reddito minimo garantito per tutte quelle persone in situazioni di necessità che non beneficiavano di alcun tipo di sussidio da parte del sistema di previdenza nazionale. Con questo intervento si attivò un effetto dimostrativo tra 1989 e 1995, quando tutte le 17 Comunidades Autónomas spagnole implementarono programmi di reddito sociale. Fino adesso, l'emulazione e l'imitazione tra queste regioni hanno contribuito a frenare la discriminazione reciproca e hanno garantito un effettivo strumento equalizzatore per le politiche sociali (Arriba e Moreno, 2005).

Nella sua fase di post-devolution, il governo scozzese lanciò nel 2002 una nuova politica di assistenza e attenzione personale gratuita per gli anziani. Il costo totale di questi sussidi ammontava a circa 1.000 milioni di sterline nel 2006. Lord Sutherland, che presiedeva la Royal Commission della Long Term Care of the Elderly nel 1999, nove anni più tardi (nel 2008) informava che questa politica aveva ricevuto un appoggio unanime: il programma era stato eseguito nel 2002 da un governo scozzese laburista/democratico liberale e aveva ricevuto un forte sostegno anche da parte del successivo governo del partito nazionalista scozzese SNP. Si calcola che 50.000 anziani hanno usufruito di questa politica sociale in Scozia. Diversamente da quanto accaduto nei Paesi Baschi, né il Galles né l'Irlanda del Nord $^{9}$ imitarono questa proposta virtuosa a causa di una questione di sostenibilità finanziaria che tale politica avrebbe rappresentato per il governo centrale della Gran Bretagna (Sutherland, 2008).

È necessaria una maggiore analisi per sistematizzare la scarsa informazione disponibile sulle iniziative regionali di welfare nell'Unione Europea e per valutare gli effetti dell'apprendimento e dell'innovazione nella politica sociale indotti dai livelli intermedi di governo. Si devono esprimere chiare avvertenze riguardo alla limitata capacità che le regioni hanno - anche per motivo delle loro dimensioni - quando devono

\footnotetext{
${ }^{9}$ Malgrado il governo dell' Irlanda del Nord abbia votato a favore del servizio gratuito di assistenza per le persone anziane, dal 2008 il Ministro della Sanità ha stabilito che la data ufficiale per l'avvio di questo programma sarebbe rimasta aperta, entrando in vigore dal 2010 o 2011. In Inghilterra e in Galles nessuno, tra laburisti e conservatori, si è dichiarato a favore dello schema scozzese per questa politica.
} 
sviluppare un welfare per conto proprio. Tra queste, con l'argomento della "corsa al ribasso" (race to the bottom), si sottolinea che la globalizzazione può contribuire a ridurre i diritti dei cittadini. Certamente, e soprattutto nei paesi meno sviluppati, le condizioni sociali e strutturali di un territorio possono essere modificate in maniera decisiva a seconda delle strategie di investimento diretto che si scelgano nei consigli di amministrazione delle imprese, spesso sulla base di interessi a breve termine oppure con l'aspettativa di benefici immediati, ignorando le necessità di medio e lungo termine delle regioni interessate. In tale circostanza, i governi locali, regionali e nazionali sarebbero disponibili ad offrire maggiori incentivi per questi investimenti sulla base di salari minori e di tutele sociali inferiori per abbassare i costi produttivi.

È pur vero che ci sono esempi dove avviene esattamente il contrario. Tuttavia, in entrambi i casi, è bene tener presente che gli attori e i decisori politici - a livello statale e sub-statale - possono proporsi e diventare creditori a seconda della loro logica situazionale e strategica (credit claimers). Il successo delle iniziative territoriali dipende, quindi, da come alcune regioni in Europa riescano a sfruttare meglio il proprio capitale umano e relazionale (Berry et al., 2003; Moreno e McEwen, 2005).

In molti casi, l'ambito regionale ha dato prova di essere un livello amministrativo dove si prendono decisioni in maniera responsabile (Goodin, 2003). Le regioni hanno anche dimostrato che l'investimento in innovazione ha superato i vantaggi del passato riferiti a élite centraliste che decidevano quali politiche territoriali realizzare e come investire il denaro pubblico. Accade spesso che quando la necessità dell'innovazione si fa più pressante cresce pure la convenienza di "laboratori di democrazia" dove il governo sub-statale e gli attori locali lavorano per promuovere nuove iniziative politiche (Donahue, 1997). Tra le varie preferenze, con rispetto alla implementazione di politiche sociali a livello regionale per far fronte ai NSR, si possono citare i seguenti criteri:

- Responsabilità (accountability), che favorisce la partecipazione democratica dei cittadini nella vita pubblica attraverso reti e coalizioni regionali e locali di governo o di gestione. Questi attori, o gruppi di attori, rispettano un criterio di prossimità e ottimizzano le risorse delle comunità locali grazie a una migliore sintonia con le loro necessità. A questo proposito, è importante che i processi di apprendimento istituzionale integrino l'articolazione delle domande sociali esistenti e il controllo dei risultati delle politiche sociali sul territorio.

- Legittimità (legitimacy), auspicata dal finanziamento dell'Unione Europea a favore delle regioni meno sviluppate. Tale principio di 
solidarietà verso i territori ed i cittadini più bisognosi è pienamente legittimato a livello comunitario dal 1975, con la creazione dei fondi strutturali di coesione per bilanciare lo sviluppo di differenti regioni europee e per ridurre le disparità socio-economiche al loro interno.

- Ottimizzazione (optimality), basata soprattutto su considerazioni di grandezza ed economia di scala rispetto alla convenienza di certe politiche sociali (per esempio i servizi di assistenza alla persona). Dalle inchieste emerge che la maggioranza dei cittadini preferisce essere assistita nei propri luoghi di residenza e, se possibile, da famigliari o vicini. Tali preferenze diventano spesso una priorità quando i costi per l'erogazione dei servizi sociali sono considerati a carico delle istituzioni regionali e locali.

- Partenariato (partnership), che si esprime come il risultato della crescente interdipendenza tra le risorse pubbliche e private in un contesto di welfare mix. Il partenariato sociale coinvolge e massimizza la consultazione, la concertazione e l'interscambio di informazione su questioni relative allo sviluppo del welfare come la promozione pubblico-privata dei diritti sociali (a questo proposito, si pensi alle disposizioni corporative sull'orario di lavoro per conciliare l'attività professionale e il tempo in famiglia).

- Riassestamento istituzionale (recalibration). Le regioni con una prospettiva di localismo cosmopolita ${ }^{10}$ potrebbero abbandonare la linea guida tradizionale dello stato centrale e i suoi meccanismi di cooptazione politica. Questa strategia si riflette sullo sviluppo del senso di comunità locale e al contempo sulla partecipazione nel contesto internazionale. C'è quindi un esteso e continuo aggiustamento tra il particolare e il generale, o meglio tra la transnazionalizzazione e la sussidiarietà. Per questa ragione, la riconfigurazione dei confini dei welfare nazionali offre opportunità inedite all'Unione Europea e alle regioni per stabilire nuovi strumenti di politica sociale.

Le visioni normative contro la decentralizzazione, in generale, e la sussidiarietà del welfare, in particolare, si riferiscono al possibile inasprimento delle disuguaglianze territoriali. Tale circostanza si verifica

${ }^{10}$ Il localismo cosmopolita si riferisce principalmente ai sistemi di governo di medie dimensioni, facendo o meno riferimento al contesto statale. In Europa tali configurazioni possono essere riscontrate non solo nei piccoli stati-nazione (Danimarca, Irlanda, Lussemburgo) e nelle minoranze nazionali senza stati (Catalogna, Fiandre, Scozia), ma anche in alcune regioni (Bruxelles, Linguadoca, Lombardia) e grandi aree metropolitane (Berlino, Londra, Madrid) (Moreno, 2005). 
spesso in quei paesi dove le politiche regionali definite dai governi nazionali di fatto "congelano" - se non incrementano - le differenze previamente esistenti. Si tratta di una forma di gestire gli interessi locali che non compete alle istituzioni regionali e che, con frequenza, oltre ad essere responsabile dell'inefficiente offerta di servizi da parte della amministrazione centrale, impedisce l'innovazione politica.

In futuro, prevedibilmente, i diritti di previdenza sociale contro i "vecchi rischi” rimarranno delle responsabilità statali e saranno finanziati da fonti contributive e fiscali di ordine nazionale. D'altra parte, la copertura dei NSR potrebbe essere ben compensata da "nuovi" incrementi finanziari in ambito regionale e locale. Per questa ragione, gli accordi per facilitare una maggiore autonomia fiscale a livello sub-statale hanno un'importanza rilevante. Le regioni che dispongono di un grado superiore di autonomia fiscale sarebbero in grado di offrire nuove risorse per lo sviluppo della cittadinanza sociale. Nella prossima sezione, attraverso la rassegna di alcune politiche di ridistribuzione, decentralizzazione e solidarietà territoriale, cercheremo di evidenziare le possibilità delle comunità substatali di formulare ed implementare nuovi interventi di welfare.

\section{Autonomia, solidarietà e redistribuzione territoriale}

La forza emotiva della nazionalità, così come la solidarietà e la mutua appartenenza che essa sostiene, serve a precisi obiettivi politici. L'idea che lo stato-nazione rappresenti un popolo che condivide un'identità comune $\mathrm{e}$ un insieme di valori civici ha tradizionalmente: (1) aumentato la sua legittimità; (2) favorito la partecipazione dei cittadini verso un processo democratico; (3) sostenuto gran parte del discorso usato per spiegare le scelte politiche dei governi. L'ingerenza dello stato in ogni tipo di questione e in tutti gli ambiti territoriali è stata spesso giustificata sulla base di una medesima identità nazionale. Per esempio, le variazioni del tipo di tassazione o delle politiche redistributive, oppure la legislazione su questioni sociali o morali possono essere giustificate in nome della comunità nazionale e dei valori che essa vuole difendere. Lo scenario diventa più articolato quando i cittadini manifestano identità multiple e una lealtà territoriale diversa. Tuttavia, non esiste alcuna contraddizione intrinseca nel legittimare una governance multilivello. Quindi, differenti proposte di politica sociale potrebbero rispondere alle domande di autonomia e solidarietà in contesti istituzionali plurali e transnazionali. 
La ridistribuzione delle risorse tra i cittadini - in maniera inter-personale ed inter-territoriale - ha avuto lo stato-nazione e le politiche di reddito come, rispettivamente, contesto e strumenti privilegiati per il raggiungimento della coesione sociale in epoca contemporanea. Durante il secolo scorso, si è cercato di articolare un welfare inclusivo alimentando una solidarietà sociale che andasse oltre le differenze di classe e mantenendo lo stato-nazione come principale arena per gestire i rischi sociali in maniera congiunta. Le alleanze dei gruppi sociali e di classe che venivano strette a livello nazionale generavano sentimenti di appartenenza ed unione. Gli attori politici regionali e locali hanno insistito, però, sul fatto che la solidarietà sociale godesse di maggiore legittimità in territori più piccoli, dove viene condiviso un forte senso identitario e di comunità (ad esempio la Catalogna, le Fiandre o la Scozia), mentre al contempo si garantiva un riferimento transnazionale per prestazioni costituzionali non discriminatorie ed egualitarie (come nel caso dell’Unione Europea) ${ }^{11}$.

In questo scenario, come entità che incorporava i sentimenti di solidarietà e reciprocità, l'identità nazionale rappresentava un requisito essenziale per il funzionamento del sistema di welfare redistributivo ${ }^{12}$. Pertanto, in linea con questo argomento, la solidarietà, la redistribuzione e un welfare unitario costituivano un circolo virtuoso per la copertura dei diritti sociali.

I paesi con una cultura politica omogenea, $\mathrm{o}$ in cui le istituzioni costituenti riconoscevano i benefici di una implementazione strategica di tipo top-down, approvavano e sostenevano i sistemi di welfare nazionali: i governi centrali si occupavano della ridistribuzione mentre le autorità substatali dovevano farsi carico delle funzioni amministrative e dell'implementazione delle politiche sociali in ambito regionale. Tuttavia,

\footnotetext{
${ }^{11}$ Negli anni novanta, la percentuale degli scozzesi di differenti classi sociali che si identificavano con altri scozzesi più che con gli inglesi della stessa classe era fortemente in aumento. La Scottish Parliamentary Election Survey del 1999 ha riscontrato che sia tra gli scozzesi che si definiscono come classe lavoratrice (71\% della popolazione totale in Scozia), sia tra quelli che si definiscono come classe media, la maggioranza si identifica con i connazionali di classe opposta (Paterson et al., 2001).

12 David Miller (1995 e 2000) si oppone all'idea di riconoscere le identità delle minoranze senza associarle a una più ampia identificazione nazionale. Allo stesso modo, Margaret Canovan (1996) sostiene che il senso della solidarietà comune associato all'identità nazionale ci fa capire perché i beni e le proprietà dovrebbero essere condivisi e definisce i limiti entro i quali dovrebbero essere redistribuiti. I processi di "demarcazione e vincolazione” (boundary-bonding) si sono sviluppati a livello nazionale nei modelli di solidarietà dei welfare (Ferrera, 2005).
} 
le evidenze empiriche che sostengono questa gestione "a somma positiva" non sono consistenti.

Dopo il periodo dei cosiddetti "trenta anni gloriosi" (1945-1975), o “epoca d' oro del welfare”, tra tutte le richieste sociali collettive, gli sviluppi politici hanno messo in primo piano le aspirazioni dei singoli territori dentro gli stati. Di pari passo con la progressiva perdita di legittimità di un modello statale di intervento verticale, la riscoperta delle identità etnoterritoriali e la ricerca di una autonomia territoriale sub-statale, l'affermarsi dei NSR ha aperto una nuova "finestra di opportunità" alle realtà regionali e locali per l'azione e l'innovazione politica. Queste iniziative si sono scontrate con i tentativi centralizzati di smantellare il welfare verticale secondo il credo neo-liberale della deregolamentazione e di "far retrocedere le frontiere dello stato" (rolling back the frontiers of the state).

Il dibattito sul fatto che la decentralizzazione obblighi o meno alla ridistribuzione è interminabile. Esiste un'ampia letteratura internazionale che impiega analisi multivariate per capire quali fattori determinano i livelli di spesa sociale (Hicks e Swank, 1992; Huber e Stephens, 2001). Si tratta di riferimenti bibliografici con una lunga tradizione (Cameron, 1978) i cui risultati spesso indicano che il federalismo e/o la decentralizzazione provocano l'espansione del welfare nazionale. Ulteriori studi segnalano che il rescaling territoriale può avere degli effetti negativi superiori a quelli di qualsiasi altra variabile istituzionale; maggiori del livello di corporativismo nelle decisioni politiche, del tipo di sistema elettorale o del sistema presidenziale di governo (Swank, 2002). Tuttavia, i paesi federali con un welfare maggiormente sviluppato, come l'Australia o il Canada, presentano una importante correlazione positiva tra la spesa sociale e la ridistribuzione (Obinger et al., 2005).

Oltre alla struttura dello stato, la ripartizione delle risorse disponibili può essere influenzata anche dalla composizione etnica di un paese. A questo proposito, si è registrato un grado di ridistribuzione più limitato in quei paesi etnicamente eterogenei o con alti tassi di immigrazione. Diversi autori hanno evidenziato che le politiche pubbliche preposte all'identificazione e all'assorbimento della diversità interna risultano controproducenti per la solidità del welfare. Questo fenomeno può avere numerose conseguenze, per esempio: (1) un effetto di crowding-out, con la deviazione di energie, tempo e risorse dalle pratiche di ridistribuzione a quelle di riconoscimento; (2) un effetto corrosivo della fiducia e della solidarietà tra i cittadini; (3) una diagnosi erronea, con soluzioni "culturaliste" che distolgono l'attenzione dal "vero problema" delle disuguaglianze di classe. D’altra parte è opportuno segnalare che la 
relazione causale tra la riduzione dei diritti di welfare e le politiche di accoglienza, identificazione e controllo non è stata corroborata da ricerche empiriche (Banting e Kymlicka, 2006).

Per le regioni dei paesi decentralizzati, la forma di devolution è un importante ambito di riferimento per valutare i risultati delle politiche sociali. Alcuni riscontri evidenziano che i paesi con una responsabilità decentralizzata di spesa, ma con una responsabilità centralizzata di aumento fiscale, a parità di condizioni tendono a spendere di più. Al contrario, in quelle realtà nazionali in cui tali responsabilità sono decentralizzate il livello di spesa è decisamente inferiore (Rodden, 2003).

L'autonomia delle voci di spesa pubblica e sociale è vista come una parte integrante dell'autonomia politica non solo dalle regioni e dalle conurbazioni più ricche (come i Paesi Baschi, il Quebec o Londra), ma anche da quelle relativamente più povere (come l'Andalusia, la Corsica o Berlino). L'autonomia si confronta anche con il principio di solidarietà inter-territoriale, che implica a livello degli stati-nazione la ridistribuzione di fondi dalle regioni ricche a quelle povere e, in un contesto più ampio come quello dell'Unione Europea, un trasferimento di risorse analogo ma tra paesi diversi. L'obiettivo finale dei sistemi di perequazione, consiste nel raggiungimento di un livello minimo di servizi basici, nell'acquisizione dei diritti di cittadinanza e in una distribuzione adeguata del carico finanziario. I trasferimenti economici in blocco sono generalmente utilizzati dai Ministeri della Finanza per mettere in pratica un certo grado di equità interterritoriale. Il dibattito sul fatto che questo finanziamento debba avere una funzione specifica o meno è cruciale per l'esercizio di una autonomia discrezionale da parte delle regioni destinatarie.

I sistemi di perequazione cercano di ridistribuire in maniera equilibrata le risorse disponibili. Con frequenza, le regioni più avanzate esprimono le proprie critiche e riserve quando ritengono che tali pratiche sono troppo generose e prive di un criterio chiaro e condiviso di distribuzione affinché le regioni più depresse (che ricevono il loro aiuto) siano motivate ad incrementare le proprie performance di sviluppo. Queste ultime, dal canto loro, richiedono invece una spesa pubblica superiore con l'obiettivo di rafforzarsi per raggiungere il livello delle altre comunità politiche.

La ridistribuzione potrebbe realizzarsi anche nella forma di investimenti pubblici statali in progetti di grandi infrastrutture. Tale opzione può risultare discrezionale oltre a discriminare e disattendere le richieste di alcuni territori per "inconfessate" ragioni di partito politico (si pensi per esempio agli investimenti improduttivi e ai trasferimenti finanziari ad personam nel mezzogiorno italiano durante il secondo dopoguerra). Queste 
dinamiche avrebbero potuto essere legittimate dall'alto appoggio politico tra regioni goduto da programmi di welfare nazionali di cui sono responsabili le autorità centrali come nel caso delle pensioni di anzianità, dei sussidi di disoccupazione e degli aiuti contro l'esclusione sociale ${ }^{13}$.

I timori che la "corsa al ribasso" (race to the bottom), oppure la conversione delle regioni e degli enti locali in "calamite" del welfare (welfare magnets), sono spesso esagerati. Sebbene i politici possano credere che sussidi generosi attraggano beneficiari del sistema di welfare da altri territori, esistono pochi riscontri a conferma di queste affermazioni. Persino in un paese federale come gli Stati Uniti - dove esiste una mobilità geografica maggiore rispetto all'Europa - le prove empiriche per poter affermare che possa davvero verificarsi una dinamica di "voto con i propri piedi" (voting with their feet) sono scarse. Data l'importanza di questa logica per la difesa normativa della ridistribuzione come una questione di competenza nazionale, riteniamo che tale prospettiva possa essere rivista. Esistono, infatti, prove empiriche che indicano una crescente aspirazione delle regioni con devolution ad assumere maggiori poteri per sviluppare ed innovare politiche di welfare a livello locale (si pensi per esempio alle province canadesi, ai Länder tedeschi, alle Regioni italiane, alle Comunidades Autónomas spagnole o alle amministrazioni con devolution in Gran Bretagna) (Moreno e McEwen, 2005).

Il processo di decentralizzazione nelle democrazie avanzate potrebbe anche modificare la tradizionale visione di welfare. L'autonomia substatale sulla legislazione del welfare può provocare divergenze politiche e distinte configurazioni del sistema sociale entro i confini di un singolo stato. Una parte importante di questo fenomeno rappresenta l'inevitabile conseguenza del riassestamento territoriale, specialmente in quei casi in cui il trasferimento di competenze amministrative è stata la risposta alle domande di auto-governo.

La divergenza nell'ambito della politica sociale è un fattore potenzialmente in grado di indebolire la solidarietà inter-regionale solo se questa suppone che i cittadini residenti in differenti regioni dello stesso stato non accedano agli stessi servizi, o non gli vengano riconosciuti gli stessi benefici e diritti. Anche se il controllo della copertura assicurativa

${ }^{13}$ Recentemente, i regionalisti delle Fiandre hanno proposto la separazione del sistema nazionale di previdenza sociale contando con l'appoggio di una maggioranza dei cittadini fiamminghi. Tale riforma è "improbabile" dal momento che richiederebbe un ampio consenso tra le due comunità (la francofona e la fiamminga) per poter cambiare la costituzione (Dandoy e Baudewyns, 2005). 
previdenziale tende ad essere una prerogativa del governo centrale, l'autonomia politica sub-statale ha supposto il decentramento di importanti aree del sistema di previdenza sociale, soprattutto con rispetto ai servizi di assistenza alle persone. La sussidiarietà del welfare può stimolare l'innovazione delle politiche pubbliche, con un "effetto dimostrativo" (demonstration effect) che minimizza gli effetti indesiderati della solidarietà nello stato-nazione. In un contesto transnazionale, l'assunzione implicita di solidarietà nelle politiche di ridistribuzione del reddito promosse dall'integrazione europea hanno ampliamente legittimato l'intervento di tipo multilivello.

\section{Conclusioni: le regioni europee come promotrici di welfare}

Dall'inizio della crisi finanziaria mondiale provocata dal collasso creditizio statunitense nel 2007, i sistema di welfare europei affrontano la sfida per il rinnovamento del patto tra il benessere sociale e la crescita economica, responsabile della inclusione dei cittadini dell'Unione e della coesione tra tutti gli stati membri. L'obiettivo non è solo preservare i diritti sociali di un modello europeo distinto rispetto, per esempio, agli Stati Uniti o alla Cina, ma di estendere la cittadinanza sociale a quegli ambiti del welfare che sono suscettibili di nuove trasformazioni sostanziali e strategiche.

Le regioni possono aspirare all'innovazione delle politiche sociali e all'espansione del welfare pur rimanendo nei rispettivi contesti nazionali ed europei di solidarietà e ridistribuzione. Considerata la natura stessa della governance multilivello, le regioni hanno pochi impedimenti per sviluppare la cittadinanza sociale, come nel caso dell'Unione Europea, con programmi basati su due premesse:

1. Negli ultimi decenni, l'Unione Europea ha abbracciato l'idea che la "regolamentazione soft" si adegui meglio alle ragioni dell'unità nella diversità, caratteristica del processo di europeizzazione e alle necessarie dinamiche di apprendimento e condivisione nelle politiche sociali. In questo senso, il Metodo Aperto di Coordinamento (MAC) può essere considerato un nuovo paradigma di regolamentazione sociale ${ }^{14}$. Il MAC

${ }^{14}$ Questo metodo consiste nelle seguenti fasi: (a) le istituzioni europee propongono una serie di linee guida, con obiettivi generali spesso sintetizzati in concetti come "attivazione" e "occupabilità"; (b) ciascun stato membro inserisce queste linee guida nei rispettivi piani programmatici nazionali (National Reform Programs); (c) si stabilisce un sistema di 
non si basa su norme dettagliate, bensì sulla istituzione di procedure generali (procedural regulation) che favoriscono una maggiore flessibilità, varietà e libertà di gestione politica. La promozione di questa forma di governance favorisce una risposta alla crisi della legittimità democratica con cui deve confrontarsi ciascun stato membro dell'Unione Europea. Questo strumento sta rimpiazzando la prospettiva centralista basata su un modello di subordinazione formale. Si tratta, quindi, di un tipo di regolamentazione che cerca il coinvolgimento di un grande numero di attori nei processi decisionali, in modo tale da far confluire un'ampia gamma di prospettive politiche e di interessi sociali.

2. Le pratiche di emulazione risultano appropriate sia per la varietà delle condizioni interne all'Unione Europea sia per l'implementazione concreta del principio di sussidiarietà, grazie anche alla condivisione di best practice. Queste ultime rappresentano degli indicatori di riferimento che vengono impiegati in una prospettiva sincronica (tra paesi) e diacronica, e che configurano il regolamento di procedura d'accordo con le preferenze di flessibilità, discrezione e varietà. L'emulazione potrebbe risultare il meccanismo più indicato per lo sviluppo regionale del welfare, sempre che non venga intesa come un processo imposto dall'esterno. La conciliazione dei diversi livelli amministrativi e delle molteplici aspettative politiche dei cittadini dovrebbero essere un fattore determinante per la costruzione dell'Europa come una comunità di confidenza mutua (community of trust).

L'incoraggiamento per la convergenza dei risultati di welfare sotto l'auspicio delle istituzioni centrali dell'Unione Europea si basa sull'idea di un modello socio-economico e di una cittadinanza europea condivisi. Gli sforzi realizzati dagli stati membri meno sviluppati e "ultimi arrivati” per il raggiungimento degli obiettivi comunitari ha favorito una maggiore coesione e credibilità per il progetto europeo. Allo stesso modo, anche le regioni e le comunità politiche sub-statali si confrontano con gli incentivi ad abbandonare la loro posizione come "ritardari" rispetto alle proprie aspirazioni di welfare e di sviluppo della cittadinanza sociale.

Negli scenari più auspicabili per il futuro si dovrebbero favorire il dispiegamento di nuove sinergie in un welfare mix di diritti, interventi e strumenti di politica sociale con effetti di lungo termine per migliorare le

indicatori per comparare i paesi dell'Unione in diversi intervalli di tempo e per identificare le best practices da tenere come punti di riferimento; (d) viene avviato un processo di valutazione, revisione e monitoraggio continuo da parte di gruppi paritetici nell'ambito comunitario. 
condizioni di vita dei cittadini. Si tratta, dunque, di un risultato in linea con la natura stessa della cittadinanza multilivello, oltre che di una opzione strategica importante per condividere e sostenere il peso della crisi nell'attuale congiuntura di instabilità economica internazionale.*

\section{Riferimenti bibliografici}

Adelantado J. e Calderón-Cuevas E. (2006). Globalization and the Welfare State: the same strategies for similar problems? Journal of European Social Policy, 16: 374-86. Doi: 10.1177/0958928706068275.

Archibugi D., Held D. e Kohler M. (a cura di) (1998). Re-imagining political community. Studies in cosmopolitan democracy. Polity Press. Cambridge.

Armingeon K. e Bonoli G. (a cura di) (2007). The politics of post-Industrial Welfare States. Adapting post-war social policies to new social risks. Routledge. New York.

Arriba A. e Moreno L. (2005). Spain: Poverty, social exclusion and safety nets. In: M. Ferrera (a cura di). Welfare State Reform in Southern Europe. Fighting poverty and social exclusion in Italy, Spain, Portugal and Greece. Routledge. London.

Banting K. e Kymlicka W. (a cura di) (2006). Multiculturalism and the Welfare State. Recognition and redistribution in contemporary democracies. Oxford University Press. New York.

Bartolini S. (2005). Restructuring Europe: Centre formation, system building and political structuring between the nation-state and the European Union. Oxford University Press. New York.

Berg L. (2007). Multi-level Europeans. The influence of territorial attachments on political trust and Welfare attitudes. Göteborgs universitet. Gothenburg.

Berry W., Fording R. e Hanson R. (2003). Reassessing the "race to the bottom" in state Welfare policy: resolving the conflict between individual level and aggregate research. Journal of Politics, 65: 327-349.

Bonoli G. (2005). The politics of the new social policies: Providing coverage against new social risks in mature welfare states. Policy and Politics, 33: 421449. Doi: 10.1332/0305573054325765.

Bruter M. (2005). Citizens of Europe? The emergence of a mass European identity. Palgrave McMillan. New York. Doi: 10.1057/9780230501539.

Cameron D. (1978). The expansion of political economy: a comparative analysis. American Political Science Review, 72: 1243-1261. Doi: 10.2307/1954537

Canovan M. (1996). Nationhood and political theory. Edward Elgar. Cheltenham.

Dahl R. (1989). Democracy and its Critics. Yale University Press. New Haven, NJ.

Dandoy R. e Baudewyns P. (2005). The preservation of social security as a national function in the Belgian federal state. In: N. McEwen e L. Moreno (a cura di). The territorial politics of welfare. London Routledge. 
Del Pino E. y Van Ryzin G. (2012). Intergovernmental Responsibility for Social Policy: An Analysis of Public Preferences in Spain. Publius. The Journal of Federalism. Doi: 10.1093/publius/pjs051.

Díez-Medrano J. e Gutiérrez P. (2003). Nested identities: national and European identity in Spain. Ethnic and Racial Studies, 24: 753-758. Doi: 10.1080/01419870120063963.

Donahue J. D. (1997). Disunited States. Basic Books. New York.

Easton D. (1965). A systems analysis of political life. Wiley. New York.

Esping-Andersen G., Gallie D., Hemerijck A. e Myles, J. (2002). Why we need a new Welfare State. Oxford University Press. New York. Doi:10.1093/0199256438.001.0001

Faist T. (2001). Social citizenship in the European Union: Nested membership. Journal of Common Market Studies, 39: 37-58. Doi: 10.1111/1468-5965.00275.

Fargion V. (2000). Timing and the development of social care services in Europe. West European Politics, 23: 59-88. Doi: 10.1080/01402380008425367.

Ferrera M. (2005). The boundaries of Welfare. European integration and the new spatial politics of social protection. Oxford University Press. New York.

Ferrera M. (2008). Solidarity beyond the nation-state? Reflections on the European experience. URGE Working paper, 2/2008. http://www.urge.it/files/papers/2_wpurge2_2008.pdf [accesso 10-12-2012].

Flora P., Kuhnle S. e Urwin D. (a cura di) (1999). State formation, nation-building and mass politics in Europe. The theory of Stein Rokkan. Oxford University Press. New York.

Gagnon A-G. e Tully J. (a cura di) (2001). Multinational democracies. Cambridge University Press. New York.

Giner S. (1994). The advent of European society. International Journal of Sociology, 24: 11-39.

Goodin R. E. (2003). Democratic accountability: The third sector and All. Hauser Center for nonprofit organizations. Working Paper, No.19. http://ssrn.com/ [accesso 10-12-2012].

Gough I. (1979). The political economy of the Welfare State. Macmillan. London.

Held D. (1991). Democracy, the Nation-State and the Global System. In: D. Held (a cura di). Political Theory Today. Polity Press. Cambridge.

Hicks A. e Swank D. H. (1992). Political institutions and Welfare spending in industrialized countries, 1960-1982. American Political Science Review, 86: 65874. Doi: 10.2307/1964129.

Hobsbawm E. (1996). Ethnicity and nationalism in Europe today. In: G. Balakrishnan (a cura di). Mapping the Nation. Verso \& New Left Review. London.

Hooghe L. e Marks G. (2001). Multi-level governance and European integration. Rowman \& Littlefield. Lanham, MD.

Huber E. e Stephens J. (2001). Development and crisis of the Welfare State: Parties and policies in global markets. University of Chicago Press. Chicago, IL. Doi: 10.7208/chicago/9780226356495.001.0001. 
Jeffery C. (2009). Devolution, public attitudes and social citizenship. In: S. L. Greer (a cura di), Devolution and social citizenship in the United Kingdom. Policy Press. Bristol. Doi:10.1332/policypress/9781847420367.003.0005.

Kazepov Y. (2008). The subsidiarization of social policies: Actors, processes and impacts, European Societies, 10: 247-273. Doi: 10.1080/14616690701835337.

Keating M. (2001). Plurinational Democracy: Stateless nations in a postsovereignty Era. Oxford University Press. New York. Doi:10.1093/0199240760.001.0001.

Kunhle S. (a cura di) (2000). The survival of the European Welfare State. Routledge. London.

Lecours A. e Moreno L. (2010). Nationalism and democracy. Dichotomies, complementarities, oppositions. Routledge. London.

Lewis-Beck M., Nadeau R. e Elias A. (2008). Economics, party and the vote: Causality issues and panel data. American Journal of Political Science, 52: 84-95. Doi: 10.1111/j.1540-5907.2007.00300.x.

Linz J. J. (1997). Democracy, multinationalism and federalism. Working Paper 1997/103. Madrid, CEACS-Instituto Juan March. http://www.march.es/ceacs/publicaciones/working/archivos/1997_103.pdf. [accesso 10-12-2012].

Loughlin J. (2007). Reconfiguring the State: Trends in territorial governance in European states. Regional and Federal Studies, 17: 385-403. Doi: 10.1080/13597560701691912.

Marks G., L. Hooghe e Schakel A. (2008). Patterns of regional authority. Regional and Federal Studies, 18: 167-181. Doi: 10.1080/13597560801979506.

Marshall T. H. (1950). Citizenship and social class and other essays. Cambridge University Press. Cambridge.

Marshall T. H. (1965). Social policy in the twentieth century. Hutchinson. London.

Miller D. (1995). On nationality. Clarendon Press. Oxford.

Miller D. (2000). Citizenship and national identity. Polity Press. Cambridge.

Moreno L. (1999). Local and global: Meso-governments and territorial identities. In: W. Safran e R. Máiz (a cura di). Identity and Territorial Autonomy in Plural Societies. Routledge. Portland, OR.

Moreno L. (2003). Europeanization, meso-governments and safety nets. European Journal of Political Research, 42: 185-199. Doi: 10.1111/1475-6765.00069-i1.

Moreno L. (2005). Multiple identities and global meso-communities. In: G. Lachapelle e S. Paquin (a cura di). Mastering Globalisation. New Sub-States' Governance and Strategies. Routledge. London.

Moreno L. (2006). Scotland, Catalonia, Europeanization and the "Moreno question”. Scottish Affairs, 54: 1-21.

Moreno L. (2007). Europeanisation, decentralisation and Welfare reform. In: J. Loughlin e K. Deschouwer (a cura di). Territorial Governance for the 21st Century. Kloninklijke Vlaamse Academie van België voor Wetenschappen en Kunsten. Bruxelles.

Moreno L. (2010). Federal democracy in plural Spain. In: M. Burgess e A-G. Gagnon (a cura di). Federal democracies. Routledge. New York. 
Moreno L. e McEwen N. (2005). Exploring the territorial politics of welfare. In: N. McEwen e L. Moreno (a cura di). The Territorial Politics of Welfare. Routledge. London.

Moreno L. e Palier B. (2005). The Europeanization of Welfare: Paradigm shifts and social policy reforms. In: P. Taylor-Gooby (a cura di). Ideas and Welfare State Reform in Western Europe. Palgrave. New York.

Norris P. (2000). Global governance and cosmopolitan citizens. In: J. S. Nye e J. D. Donahue (a cura di). Governance in a Globalizing World. Brookings Institution Press. Washington, DC.

Obinger H., Leibfried S. e Castles F. (2005). Federalism and the Welfare State. New world and European experiences. Cambridge University Press. Cambridge. Doi: 10.1017/CBO9780511491856.

O’Connor J. (1973). The fiscal crisis of the State. St Martin's Press. New York.

Offe C. (1984). Contradictions of the Welfare State. Hutchinson. London.

Piattoni S. (2010). The theory of multilevel governance: Conceptual, empirical, and normative challenges. Oxford University Press. New York. Doi: 10.1093/acprof:oso/9780199562923.001.0001.

Pierson P. (2001). The New Politics of the Welfare State. New York: Oxford University Press. Doi: 10.1093/0198297564.001.0001.

Rodden J. (2003). Reviving Leviathan: fiscal federalism and the growth of government. International Organization, 57: 695-729. Doi:10.1017/S0020818303574021.

Somerville P. (2004). State rescaling and democratic transformation. Space and Polity, 8: 137-156. Doi: 10.1080/1356257042000273931.

Sutherland S. (2008). Free personal and nursing care in Scotland. European View, 7: 297-302. Doi: 10.1007/s12290-008-0046-6.

Swank D. (2002). Global capital, political institutions and political change in developed Welfare States. Cambridge University Press. Cambridge. Doi: 10.1017/CBO9780511613371.

Taylor-Gooby P. (a cura di) (2001). Welfare States under pressure. Sage. London.

Taylor-Gooby P. (2004). New risks and social change. In: P. Taylor-Gooby (a cura di). New Risks, New Welfare: The Transformation of the European Welfare. Oxford University Press. New York. Doi: 10.1093/019926726X.003.0001

Van Hecke S. (2003). The principle of subsidiarity: Ten years of application in the European Union. Regional and Federal Studies, 13: 55-80. Doi: 10.1080/714004786. 